\title{
Pulsating Variable Stars in the Coma Berenices dwarf spheroidal galaxy $^{1}$
}

\author{
Ilaria Musella ${ }^{2}$, Vincenzo Ripepi ${ }^{2}$, Gisella Clementini ${ }^{3}$, Massimo Dall'Ora $^{2}$, Karen \\ Kinemuchi ${ }^{4}$, Luca Di Fabrizio ${ }^{5}$, Claudia Greco ${ }^{6}$, Marcella Marconi ${ }^{2}$, Horace A. Smith ${ }^{7}$, \\ Mario Radovich ${ }^{2}$ and Timothy C. Beers ${ }^{8}$
}

\begin{abstract}
We present $B, V, I$ time-series photometry of the Coma Berenices dwarf spheroidal galaxy, a faint Milky Way satellite, recently discovered by the Sloan Digital Sky Survey. We have obtained $V, B-V$ and $V, V-I$ color-magnitude diagrams that reach $V \sim 23.0-23.2$ mag showing the galaxy turnoff at $V \sim 21.7$ mag, and have performed the first study of the variable star population of this new Milky Way companion. Two RR Lyrae stars (a fundamental-mode -RRaband a first overtone -RRc- pulsator) and a short period variable with period $P=0.12468$ days were identified in the galaxy. The RRab star has a rather long period of $P_{a b}=0.66971$ days and is about 0.2 mag brighter than the RRc variable and other non-variable stars on the galaxy horizontal branch. In the period-amplitude diagram the RRab variable falls closer to the loci of Oosterhoff
\end{abstract}

\footnotetext{
${ }^{1}$ Based on data collected at the $1.52 \mathrm{~m}$ telescope of the INAF-Osservatorio Astronomico di Bologna, Loiano, Italy, at the $2.3 \mathrm{~m}$ telescope at the Wyoming Infrared Observatory (WIRO) at Mt. Jelm, Wyoming, USA, and at $2.5 \mathrm{~m}$ Isaac Newton Telescope, La Palma, Canary Island, Spain.

${ }^{2}$ INAF, Osservatorio Astronomico di Capodimonte, Napoli, Italy, ilaria@na.astro.it, ripepi@na.astro.it, dallora@na.astro.it, marcella@na.astro.it,radovich@na.astro.it

${ }^{3}$ INAF, Osservatorio Astronomico di Bologna, Bologna, Italy; gisella.clementini@oabo.inaf.it

${ }^{4}$ Universidad de Concepción, Departamento de Física, Concepción, Chile, and University of Florida, Department of Astronomy, Gainesville, FL 32611-2055, USA; kkinemuchi@astro-udec.cl

${ }^{5}$ INAF, Centro Galileo Galilei \& Telescopio Nazionale Galileo, S. Cruz de La Palma, Spain; difabrizio@tng.iac.es

${ }^{6}$ Observatoire de Geneve, Sauverny, Switzerland; claudia.greco@unige.ch

${ }^{7}$ Department of Physics and Astronomy, Michigan State University, East Lansing, MI 48824-2320, USA; smith@pa.msu.edu

${ }^{8}$ Department of Physics and Astronomy, CSCE, Center for the Study of Cosmic Evolution, and JINA, Joint Institute for Nuclear Astrophysics, Michigan State University, East Lansing, MI 48824, USA; beers@pa.msu.edu
} 
type-II systems and evolved fundamental-mode RR Lyrae stars in the Galactic globular cluster M3. The average apparent magnitude of the galaxy horizontal branch, $\left\langle V_{\mathrm{HB}}\right\rangle=18.64 \pm 0.04 \mathrm{mag}$, leads to a distance modulus for the Coma dSph $\mu_{0}=18.13 \pm 0.08 \mathrm{mag}$, corresponding to a distance $d=42_{-1}^{+2} \mathrm{kpc}$, by adopting a reddening $E(B-V)=0.045 \pm 0.015 \mathrm{mag}$ and a metallicity $[\mathrm{Fe} / \mathrm{H}]=-2.53 \pm 0.05$ dex.

Subject headings: galaxies: dwarf - galaxies: individual (Coma) —stars: distances — stars: variables: other - techniques: photometric

\section{Introduction}

Over the past few years the analysis of the Sloan Digital Sky Survey (SDSS) data led to the discovery of several ultra-faint companions of the Milky Way (MW). The new systems include two very faint globular clusters (GCs): Koposov 1 and 2 (Koposov et al. 2007), and fourteen dwarf spheroidal (dSph) galaxies: Willman I, Ursa Major I, Canes Venatici I (CVnI), Ursa Major II (UMa II), Bootes I, Coma Berenices (Coma), Segue I, Canes Venatici II (CVn II), Leo IV, Hercules, Leo T, Bootes II, Leo V, and Bootes III (Willman et al. 2005a.b; Zucker et al. 2006a; Grillmair 2006; Zucker et al. 2006b; Belokurov et al. 2006, 2007; Irwin et al. 2007; Walsh et al. 2007; Belokurov et al. 2008; Grillmair 2008). The new dSphs have half-light radii similar to those of the classical MW dSph companions, however, they are much fainter, with typical effective surface brightnesses in the range from 28 to 31 $\operatorname{arcsec}^{-2}$ (Belokurov et al. 2007, hereafter B07). The new discoveries along with the 10 previously known MW dSph companions (Draco, Ursa Minor, Fornax, Carina, Sculptor, Leo I, Leo II, Sextans, Sagittarius and Canis Major; Mateo 1998; Ibata, Gilmore, \& Irwin 1995; Martin et al. 2004), bring to twenty-four the number of dSph galaxies presently known to surround the MW. In the absolute magnitude versus half-light radius plane the new dSphs are well separated from both GCs and traditional dSph galaxies, since they are generally more extended than GCs and much fainter than the old dSphs (see Fig. 8 of B07). Only CVnI, the brightest of the new SDSS dSphs, lies on the faintest tail of the distribution for traditional dSphs. All the new SDSS dSphs host a metal-poor and ancient stellar component, with metallicity generally below $[\mathrm{Fe} / \mathrm{H}] \sim-2$ dex and down to as low as $[\mathrm{Fe} / \mathrm{H}] \sim-3$

$\operatorname{dex}($ Munoz et al. 2006; Simon \& Geha 2007; Geha et al 2008; Kirby et al. 2008), and age as old as the stars in Galactic GCs like M92 (see B07). The density contours are irregular since most of these galaxies are in process of disruption. The closest ones (Bootes I, UMa II and Coma) are more distorted owing to the tidal interaction with the MW (Belokurov et al. 2006, 2007; Zucker et al. 2006b); others appear to be connected with tidal streams. With 
the exception only of Leo T, the new systems all have globular-cluster-like CMDs, showing main sequences, turn-offs, as well as hints of red giant and horizontal branches. Variable stars have been identified so far in three of the new systems, namely Bootes I, CVn I, and CVnII. Bootes I (Dall'Ora et al. 2006; Siegel 2006) and CVn II (Greco et al. 2008) both were found to contain RR Lyrae stars with properties resembling those of Oosterhoff-type II Oosterhoff 1939) GCs in the MW. Confirming its similarity to the traditional dSphs, CVn I (Kuehn et al. 2008) hosts, instead, RR Lyrae stars with properties intermediate between the two Oosterhoff types, along with a few candidate Anomalous Cepheids.

The Coma galaxy $\left(R . A .=12^{\mathrm{h}} 26^{\mathrm{m}} 59.0^{\mathrm{s}}, D E C=+23^{\circ} 54^{\prime} 15^{\prime \prime}, \mathrm{J} 2000.0, l=241.9^{\circ}, b=83.6^{\circ}\right.$, B07), located at a heliocentric distance of $44 \pm 4 \mathrm{kpc}$ with a position angle of $120^{\circ}$ and an absolute magnitude of $M_{V}=-3.6 \pm 0.6 \mathrm{mag}$, is among the faintest of the SDSS new discoveries. It has half light radii: $r_{h}($ Plummer $)=5.0$ arcmin, and $r_{h}($ exponential $)=5.9$ arcmin, respectively, and an irregular and extended shape (B07). The $i, g-i$ CMD obtained by B07 goes down to $i \sim 25 \mathrm{mag}$, is consistent with a single, old stellar population of metallicity $[\mathrm{Fe} / \mathrm{H}] \sim-2$, and shows a poorly populated horizontal branch (HB). Metallicities of radial velocity members of the Coma dSph were obtained by Simon \& Geha (2007) and confirm the low metal abundance of the galaxy. These metallicities were re-evaluated by Kirby et al. (2008), who derived for the galaxy an average metallicity $\langle[\mathrm{Fe} / \mathrm{H}]\rangle=-2.53 \pm 0.05$ dex with dispersion $\sigma_{[\mathrm{Fe} / \mathrm{H}]}=0.45$ dex.

In this Letter we present the first study of the variable stars in the Coma dSph, which led us to identify two RR Lyrae stars and one short period variable in the galaxy. We provide multiband light curves and properties of the variables, and use the magnitude of the HB stars to measure the distance to Coma. We also present $B, B-V$ and $V, V-I$ CMDs to $V \sim 23.0-23.2 \mathrm{mag}$, which show the galaxy main sequence turnoff at $V \sim 21.7 \mathrm{mag}$, and reveal the presence of several candidate blue straggler stars (BSSs).

\section{Observations and Data Reduction}

Time-series $B, V, I$ photometry of the Coma dSph galaxy was collected on 2007, March 12-16 and April 13-17, with BFOSC at the $1.52 \mathrm{~m}$ telescope of the Bologna Observatory in Loiand 1 ( $V$ and $I$ data), on April 30 and May 9-10, using WIRO-Prime, the prime focus CCD camera (Pierce \& Nations 2002) of the $2.3 \mathrm{~m}$ Wyoming Infrared Observatory telescope (WIRO) ( $B$ and $V$ data), and $B, V, I$ observations were gathered on April 2122, using WFC (Wide Field Camera), the prime focus mosaic CCD camera of the $2.5 \mathrm{~m}$

\footnotetext{
${ }^{1}$ http://www.bo.astro.it/loiano/index.htm
} 
Isaac Newton Telescope (INT). The field of view (FOV) covered by the three instruments is $13 \times 12.6 \operatorname{arcmin}^{2}$ for BFOSC@LOIANO, $17.8 \times 17.8 \operatorname{arcmin}^{2}$ for WIRO, and $33 \times 33$ $\operatorname{arcmin}^{2}$ for WFC@INT. We needed two partially overlapping fields to cover the galaxy with BFOSC, while just one WIRO pointing was sufficient, and from the WFC@INT field we could also infer some additional information about the contamination by field stars and background galaxies. We obtained $14 \mathrm{~B}$ images, $83 \mathrm{~V}$ frames and $64 \mathrm{I}$ frames of the Coma dSph. Images were pre-reduced following standard procedures (bias subtraction and flatfield correction) with IRAF. The $I$-band images were corrected for fringing by adopting, for each instrument, a well-suited fringing map. We then performed PSF photometry using the DAOPHOT IV/ALLSTAR/ALLFRAME packages (Stetson 1987, 1994). Typical internal errors of the single-frame photometry for stars at the HB magnitude level are of about $0.005 \mathrm{mag}$ in all the three bands. The absolute photometric calibration was derived using observations of standard stars in the Landolt fields SA 101, SA 107, SA 110 and PG1323

(Landolt 1992), as extended by P.B. Stetson2, which were obtained at the INT during the night of 2007, April 22. Errors of the absolute photometric calibration are $\sigma_{B}=0.01$, $\sigma_{V}=0.01$ and $\sigma_{I}=0.02 \mathrm{mag}$, respectively.

\section{Identification of the Variable Stars}

Variable stars were identified using the $V$ and $I$ time-series data, separately. First we calculated the Fourier transforms (in the Schwarzenberg-Czerny 1996 formulation) of the stars having at least 12 measurements in each photometric band, then we averaged these transforms to estimate the noise and calculated the signal-to-noise ratios. Results from the $V$ and $I$ photometries were cross-correlated, and all stars with $\mathrm{S} / \mathrm{N}>4$ in both photometric bands were visually inspected, for a total of about 500 candidates. We also checked whether some of the stars in the BSSs region might be pulsating variables of SX Phoenicis (SX Phe) type. Study of the light curves and period derivation were carried out using GRaTiS (Graphical Analyzer of Time Series, Clementini et al. 2000). We confirmed the variability and obtained reliable periods and light curves for 2 RR Lyrae stars: 1 fundamental-mode (RRab) variable with period $P=0.66971$ days (V1), 1 first-overtone (RRc) pulsator with period $P=0.31964$ days (V2), and for one short period variable with period $P=0.12468$ days (V3). Identification and properties of the confirmed variable stars are summarized in Table 1, their light curves are shown in Fig 1. Time-series data for the variable stars are available on request from the first author. Both the short period variable and the RRc star lie inside the half-light radius of the Coma galaxy. The RRab lies, instead, slightly outside

\footnotetext{
${ }^{2}$ see http://cadcwwwdao.nrc.ca/standards.
} 
this region. In the $\mathrm{CMD}$, the short period variable is located in the region of the BSSs, thus suggesting it might be an SX Phe star. However, the star does not fit the SX Phe periodluminosity relations (e.g. Santolamazza et al. 2001, Poretti et al. 2008) unless a pulsation period of one third the value supported by the period search analysis is adoped. The shape of the light curve and the similarity of the $A_{V}, A_{I}$ amplitudes may suggest a classification as a W UMa binary system. However, the present data do not allow us to definitely assess the actual nature of the star.

The two RR Lyrae stars both fall near the HB of the Coma dSph. However, while the average magnitude of the RRc star is fully consistent with the average luminosity of the HB we infer, by fitting the galaxy CMD with the ridge lines of the Galactic GC M 68 (see Section 4), that the RRab star is about $0.2 \mathrm{mag}$ brighter. The overluminosity of the RRab star could be due to geometrical projection effects, since the galaxy appears to be elongated due to the tidal interaction with the MW (see Dall'Ora et al. 2008 and Section 4). Contamination by an unresolved companion may also be possible, but this is constrained by the large amplitude of the RRab star, and by its color, which is consistent with the expected color of an RRab star. A further possibility is that evolution off the zero-age HB contributes to the enhancement of the brightness of the RRab star (see end of Section 4).

The rather long period of the RRab star suggests that Coma is, like CVn II (Greco et al. 2008), more similar to Oosterhoff type-II systems in the MW, and differs from CVn I, the brightest of the SDSS dSph's, which has Oosterhoff-intermediate type (Kuehn et al. 2008). Fig 2 shows the position of the Coma RR Lyrae stars in the $V$-band period-amplitude diagram of the Bootes I and CVn II RR Lyrae stars. The Coma RRab star (filled circle) falls closer to the loci of Oo II systems (from Clement \& Rowe 2000, dashed-dotted line) and evolved fundamental-mode RR Lyrae stars in the Galactic GC M3 (from Cacciari et al. 2005). The RRc star falls at the short extreme of the bell-shaped distribution defined by OoII RRc stars. Both these results suggest an OoII classification for the Coma dSph, although with some caution given the very small number of variable stars. We have used the parameters of the Fourier decomposition of the $V$-band light curves, along with Jurcsik \& Kovács (1996) and Morgan, et al. (2007) formulas, to estimate individual metallicities for the Coma RR Lyrae stars, on the Zinn \& West (1984) metallicity scale. Results are summarized in the last column of Table 1, they confirm the low metallicity derived for the Coma dSph by the spectroscopic studies. 


\section{CMD, Structure and distance}

The upper panels of Fig. 3 show the $V, B-V$ and $V, V-I$ CMDs of the Coma dSph. We have plotted all stellar-like objects (selected using the $C H I$ and $S H A R P$ parameters provided by the reduction packages), over the whole 0.5 square degrees covered by the INT observations. This selection is rather secure for magnitudes above $V \sim 20.5$-21.0 mag, while it becomes increasingly uncertain at fainter magnitudes. The variable stars (filled triangles) are plotted according to their intensity-averaged magnitudes and colors (see Table 1). The CMDs reach $V \sim 23.2 \mathrm{mag}$, and appear to be heavily contaminated at every magnitude level by field objects belonging to the MW halo and disk, and by galaxies which escaped selection. We have used the mean ridge lines of the Galactic globular cluster M68 (NGC 4590; solid blue lines in Fig. 3), drawn from Walker's (1994) $B, V, I$ photometry for the cluster, shifted by $\Delta V=+2.95 \mathrm{mag}$ in magnitude, and $\Delta(B-V)=+0.025 \mathrm{mag}, \Delta(V-I)=+0.030 \mathrm{mag}$ in color, to match the horizontal and red giant branches of the Coma dSph. Then we selected as most likely belonging to the galaxy the sources lying within $\pm 0.06 \mathrm{mag}$ in $B-V$, and \pm 0.05 mag in $V-I$, from the ridge lines of M 68 (red dots in the upper panels of Fig. 3 ). The slightly larger range adopted for the $B-V$ color takes into account the larger uncertainty caused by the small number of $B$ frames. To allow for the larger photometric errors below $V=21.5 \mathrm{mag}$, we also considered as belonging to the galaxy stars with colors in the range from $\pm 0.05 / 0.06$ to $\pm 0.1 \mathrm{mag}$ from the ridge lines of M 68 (cyan dots). M 68 is well suited for identifying members of the Coma dSph since, like Coma, is very metal poor $\left([\mathrm{Fe} / \mathrm{H}]_{\mathrm{M} 68}=\right.$ -2.1 dex, Walker 1994) and has a well defined and tight red giant branch, as well as an extended HB well populated inside and to the blue of the RR Lyrae instability strip (Walker 1994). Adopting for M 68 the reddening value of $E(B-V)=0.07 \pm 0.01 \mathrm{mag}$ (Walker 1994) the color shifts needed to match the horizontal and red giant branches of Coma imply a reddening of $E(B-V)=0.045 \pm 0.015$ mag for the galaxy, which can be compared with the value of $0.019 \pm 0.026 \mathrm{mag}$ derived from the Schlegel et al. (1998) maps. We also show in Fig. 3 results of the fit based on a less metal poor globular cluster: M 3 (Ferraro et al. 1997, Johnson \& Bolte 1998). The red giant branch of M 3 is too red and, to match that of Coma, would require for the galaxy a negative reddening $E(B-V) \sim-0.06$ mag. With the help of the M 68 ridgelines it is possible to determine the average luminosity of the Coma dSph $\mathrm{HB}$ roughly at the center of the RR Lyrae instability strip: $\left\langle V_{\mathrm{HB}}\right\rangle=18.64 \pm 0.04 \mathrm{mag}$, and to locate the galaxy main sequence turn-off at $V \sim 21.7 \mathrm{mag}$. The CMDs also show several stars in the BSS region (green dots in Fig. 3), which are likely BSSs of the Coma dSph. Our identification of the Coma member stars is confirmed in an excellent way by the comparison

with the Kirby et al. (2008) spectroscopic study of 24 stars with membership to the Coma dSph confirmed by radial velocity measurements (open circles in Fig. 3). The agreement between the two studies is impressive, and proves the reliability of our procedure to select 
member stars, and our identification of the Coma HB. The lower panels of Fig. 3 show the position of the stars we consider to belong to the Coma galaxy in the FOV of the $B$-band (left panel) and $V$-band (right panel) INT observations. Symbols and color-coding are the same as in the top-panels of the figure. Symbol-sizes are proportional to the object's magnitudes. The HB stars are outlined by squares. It is noteworthy that, with the exception only of the RRc variable, all the HB stars fall outside the galaxy half-light radius, but generally close to stars whose membership to Coma is confirmed by the spectroscopic studies.

A further feature is visible in the CMDs of the Coma dSph (see upper panels of Fig. 3), formed by sources having roughly $20.6<V<21.9 \mathrm{mag}, 1.3<V-I<1.5 \mathrm{mag}$, and $B-V \sim 1.5 \mathrm{mag}$. Among them those marked by black dots are mainly concentrated in the left-hand portion of the maps outlined by dashed circles (see lower panels of Fig. 3). In the literature, at this position, we find the cluster of galaxies MaxBCG J186.85861+2380004 (Koester et al. 2007), which has a photometric redshift $z=0.243050$. The feature we see in the CMDs is, probably, the "red sequence" of this cluster, as confirmed by the $V-I$, $B-V$ colors we derive for early-type galaxies at $\mathrm{z} \sim 0.25$ using the GISSEL03 spectral code (Bruzual \& Charlot 2003).

The average magnitude of the Coma dSph HB, set by the matching with the ridge lines of $\mathrm{M} 68$, is $\left\langle V_{\mathrm{HB}}\right\rangle=18.64 \pm 0.04 \mathrm{mag}$ (where the error includes both uncertainties in the photometry and in the matching procedure). Assuming $M_{V}=0.59 \pm 0.03 \mathrm{mag}$ for the absolute magnitude of HB stars at $[\mathrm{Fe} / \mathrm{H}]=-1.5$ dex (Cacciari \& Clementini 2003), $\Delta M_{V} / \Delta[F e / H]=0.214$ $( \pm 0.047) \mathrm{mag} / \mathrm{dex}$ for the slope of the luminosity-metallicity relation of the RR Lyrae stars (Clementini et al. 2003), $E(B-V)_{\text {Coma }}=0.045 \pm 0.015 \mathrm{mag}$, and $[\mathrm{Fe} / \mathrm{H}]_{\mathrm{Coma}}=-2.53 \pm 0.05$ dex (Kirby et al. 2008), the distance modulus of Coma is $18.13 \pm 0.08 \mathrm{mag},\left(d=42_{-1}^{+2} \mathrm{kpc}\right)$. Errors include uncertainties in the photometry, reddening, metallicity, and RR Lyrae absolute magnitude. On the same assumptions, the average apparent magnitude of the RRab star $\langle V\rangle=18.44 \pm 0.03 \mathrm{mag}$ leads, instead, to the distance modulus of $17.93 \pm 0.08 \mathrm{mag}$, $\left(d=39_{-2}^{+1} \mathrm{kpc}\right)$. While the first estimate is in good agreement with the distance of $44 \pm 4 \mathrm{kpc}$ found by B07, the value inferred from the RRab stars is, respectively, 3 and $5 \mathrm{kpc}$ shorter than the galaxy distance derived in the present study and in B07. An additional distance estimate for the two RR Lyrae stars was obtained using the empirical Wesenheit relation in the $B, V$ bands as defined by Kovács and Walker (2001) and calibrated by Kovács (2003, this calibration is consistent with a distance modulus for the Large Magellanic Cloud of $18.55 \mathrm{mag})$. The resulting moduli: $\mu_{0}\left(\mathrm{RR}_{\mathrm{ab}}\right)=18.06 \pm 0.10$ and $\mu_{0}\left(\mathrm{RR}_{\mathrm{c}}\right)=18.09 \pm 0.10 \mathrm{mag}$, are in good agreement with each other and are consistent with the distance inferred from the average magnitude of the galaxy HB. The overluminosity of the RRab reflects into the Wesenheit index, however it is balanced by the star longer period, thus the resulting distance modulus is in good agreement with that derived for the RRc variable. These results 
suggest that the RRab star is brighter because it has evolved off the zero age HB, rather than because it is significantly closer to us.

\section{Summary and conclusions}

We have identified and obtained $V, I$ light curves for $2 \mathrm{RR}$ Lyrae (1 RRc and 1 RRab) and one short period variable, in the Coma dSph galaxy. The behavior of the RR Lyrae stars suggests an Oosterhoff II classification for the galaxy. From the average luminosity of the HB stars, the distance modulus of the Coma dSph is $\mu_{0}=18.13 \pm 0.08 \mathrm{mag}\left(d=42_{-1}^{+2} \mathrm{kpc}\right)$. The galaxy appears to be elongated with irregular and extended shape, likely caused by the tidal interaction with the MW. The RRab star lies outside the galaxy half-light radius and is about $0.2 \mathrm{mag}$ brighter than the average magnitude of the galaxy's HB. Its bright mean magnitude leads to a distance of $d=39_{-2}^{+1} \mathrm{kpc}$, which, if interpreted in terms of projection effects, could provide a rough estimate for the galaxy elongation. However, the Wesenheit results suggest that the star overluminosity is indeed due, at least in part, to evolution off the zero-age HB. Finally, from the analysis of the CMDs we identify in the lower north-east field of the Coma galaxy, the background cluster of galaxies MaxBCG J186.85861+2380004 (Koester et al. 2007).

We warmly thank Dr Evan Kirby for sending us the identification and individual metallicities for member stars of the Coma dSph galaxy, Dr Amata Mercurio for advice with the identification of the galaxy cluster, and the anonymous referee for helpful comments. Financial support for this study was provided by Regione Campania 2003 (PI J. Alcalà) and by PRIN-INAF 2006 (PI G. Clementini). HAS thanks the US NSF for support under grant AST 0607249.

\section{REFERENCES}

Belokurov, V., et al. 2006, ApJ, 647, L111

Belokurov, V., et al. 2007, ApJ, 654, 897

Belokurov, V., et al. 2008, ApJ, 686, L83

Bruzual G., \& Charlot, S. 2003, MNRAS, 344, 1000

Cacciari, C., \& Clementini, G. 2003, in Stellar Candles for the Extragalactic Distance Scale, ed. D. Alloin \& W.Gieren (Berlin: Springer), 105 
Cacciari, C., Corwin, T. M., \& Carney, B. W. 2005, AJ, 129, 267

Clement, C.M., \& Rowe, J. 2000, AJ, 120, 2579

Clementini, G., et al. 2000, AJ, 120, 2054

Clementini, G., Gratton, R.G., Bragaglia, A., Clementini, G., Carretta, E., Di Fabrizio, L. \& Maio, M. 2003, AJ, 125, 1309

Dall'Ora, M., et al. 2006, ApJ, 653, L109

Dall'Ora, M., et al. 2008, MSAIt, 79, 710

Ferraro, F.R., Carretta, E., Corsi, C.E., Fusi Pecci, F., Cacciari, C., Buonanno, R., Paltrinieri, B., Hamilton, D. 1997, A\&A, 320, 757

Johnson, J.A., \& Bolte, M. 1998, ApJ, 115, 693

Jurcsik, J., \& Kovács, G. 1996, A\&A, 312, 111

Geha, M., Willman, B., Simon, J.D., Strigari, L.E., Kirby, E.N., Law, D.R., \& Strader, J. 2008, ApJ, in press, (arXiv:0809.2781)

Greco, C., et al. 2008, ApJ, 675, L73

Grillmair, C.J. 2006, ApJ, 645, L37

Grillmair, C.J. 2008, ApJ, in press, (arXiv:0811.3965)

Ibata, R.A., Gilmore, G., \& Irwin, M.J. 1995, MNRAS, 277, 781

Irwin, M.J., et al. 2007, ApJ, 656, L13

Landolt, A.U. 1992, AJ, 104, 340

Kirby, E.N., Simon, J.D., Geha, M., Guhathakurta, P. \& Frebel, A. 2008, ApJ, 685, L43

Koester, B. P., et al. 2007, ApJ, 660, 239

Koposov, S., et al. 2007, ApJ, 669, 337

Kovács, G., \& Walker, A.R. 2001, A\&A, 374, 264

Kovács, G. 2003, MNRAS, 342, L58

Kuehn, C., et al. 2008, ApJ, 674, L1381 Marconi, M. 2007, AJ, 133, 2752 
Martin, N.F., Ibata, R.A., Bellazzini, M., Irwin, M.J., Lewis, G.F., \& Dehnen, W. 2004, MNRAS, 348, 12

Mateo, M.L. 1998, ARA\&A, 36, 435

Morgan, S.M., Wahl, J.N., \& Wieckhorst, R.M. 2007, MNRAS, 374, 1421

Munoz, R.R., Carlin, J.L., Frinchaboy, P/M., Nidever, D.L., Majewski, S.R., \& Patterson, R. 2006, ApJ, 650, L51

Oosterhoff, P. Th. 1939, Observatory, 62, 104

Pierce, M.J., \& Nations, H.L. 2002, BAAS, 34, 749

Poretti, E., et al. 2008, ApJ, 685, 947

Santolamazza, P., Marconi, M., Bono, G., Caputo, F., Cassisi, S., \& Gilliland, R.L. 2001, ApJ, 554, 1124

Schlegel, D.J., Finkbeiner, D.P., \& Davis, M. 1998, ApJ, 500, 525

Schwarzenberg-Czerny, A. 1996, ApJ, 460, L107

Siegel, M.H. 2006, ApJ, 649, L83

Simon, J.D., \& Geha, M. 2007, ApJ, 670, 313

Stetson, P.B. 1987, PASP, 99, 191

Stetson, P.B. 1994, PASP, 106, 250 ApJ, 688, 277

Walker, A.R. 1994,AJ, 108, 555

Walsh, S. M., Jerjen, H., \& Willman, B. 2007, ApJ, 662, L83

Willman, B., et al. 2005a, AJ, 129, 2692

Willman, B., et al. 2005b, ApJ, 626, L85

Zinn, R., \& West, M.J. 1984, ApJS, 55, 45

Zucker, D.B., et al. 2006a, ApJ, 643, L103

Zucker, D.B., et al. 2006b, ApJ, 650, L41 
Table 1: Identification and properties of variable stars in the Coma dSph galaxy

\begin{tabular}{|c|c|c|c|c|c|c|c|c|c|c|}
\hline Name & $\begin{array}{c}\alpha \\
(2000)\end{array}$ & $\begin{array}{c}\delta \\
(2000)\end{array}$ & Type & $\begin{array}{c}P \\
\text { (days) }\end{array}$ & $\begin{array}{l}\operatorname{Epoch}(\max ) \\
(-2450000)\end{array}$ & $\begin{array}{c}\langle V\rangle \\
(\mathrm{mag})\end{array}$ & $\begin{array}{c}\langle I\rangle \\
(\mathrm{mag})\end{array}$ & $\begin{array}{c}A_{V} \\
(\mathrm{mag})\end{array}$ & $\begin{array}{c}A_{I} \\
(\mathrm{mag})\end{array}$ & $\underset{\mathrm{a}}{[\mathrm{Fe} / \mathrm{H}]_{\mathrm{ZW}}}$ \\
\hline V1 & 122733.50 & 235455.7 & RRab & 0.66971 & 4171.583 & 18.44 & 17.74 & 0.78 & 0.53 & -2.068 \\
\hline V3 & 122658.68 & 235704.5 & Short period & 0.12468 & 4171.950 & 21.57 & 21.13 & 0.29 & 0.28 & $\ldots$ \\
\hline
\end{tabular}

${ }^{a}$ Metallicities derived from the Fourier parameters of the light curves.
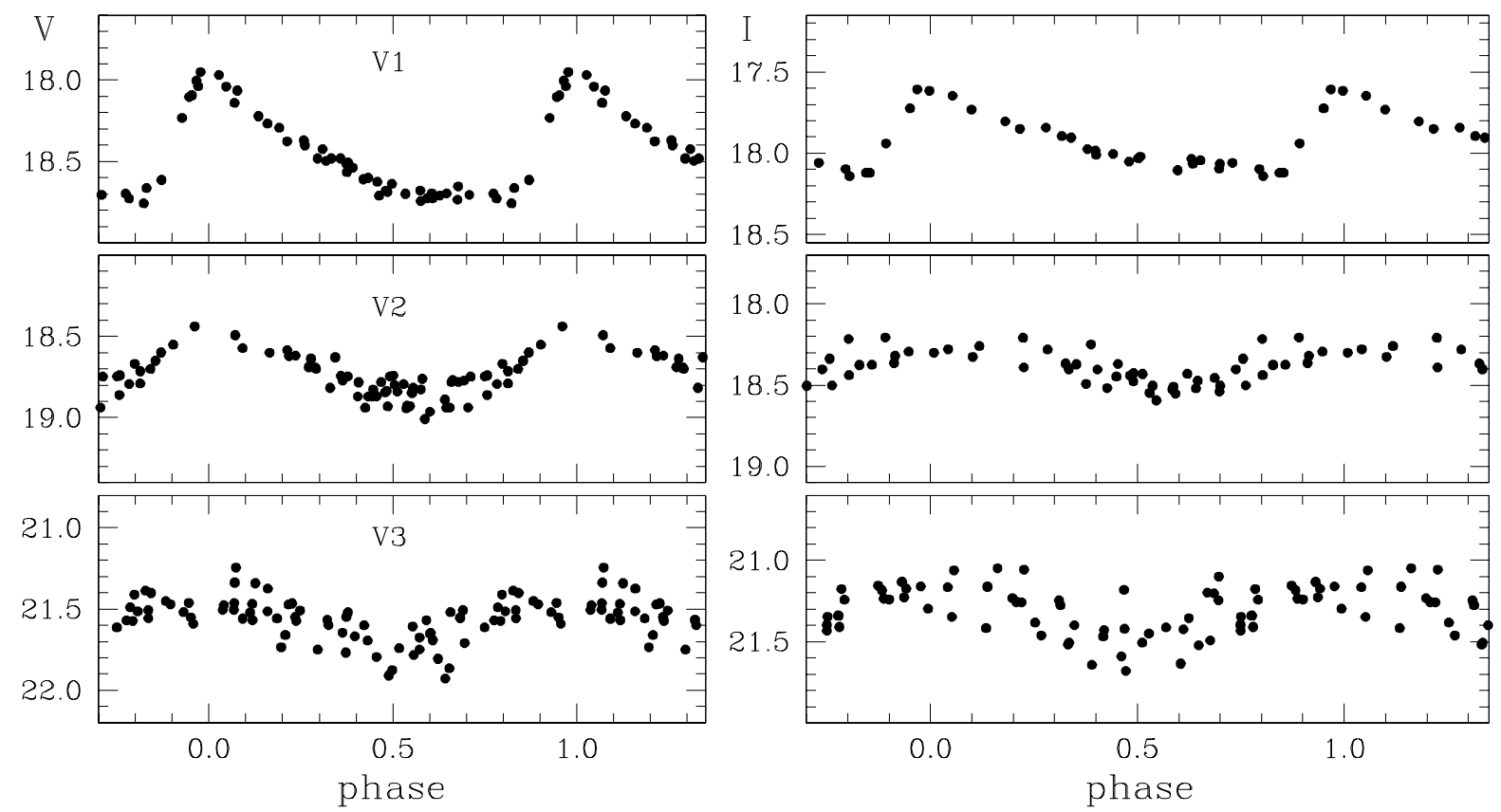

Fig. 1.- $V$ (left panels) and $I$ (right panels) light curves of variable stars discovered in the Coma dSph. From top to bottom: fundamental-mode RR Lyrae star, first-overtone RR Lyrae pulsator, short period variable. 


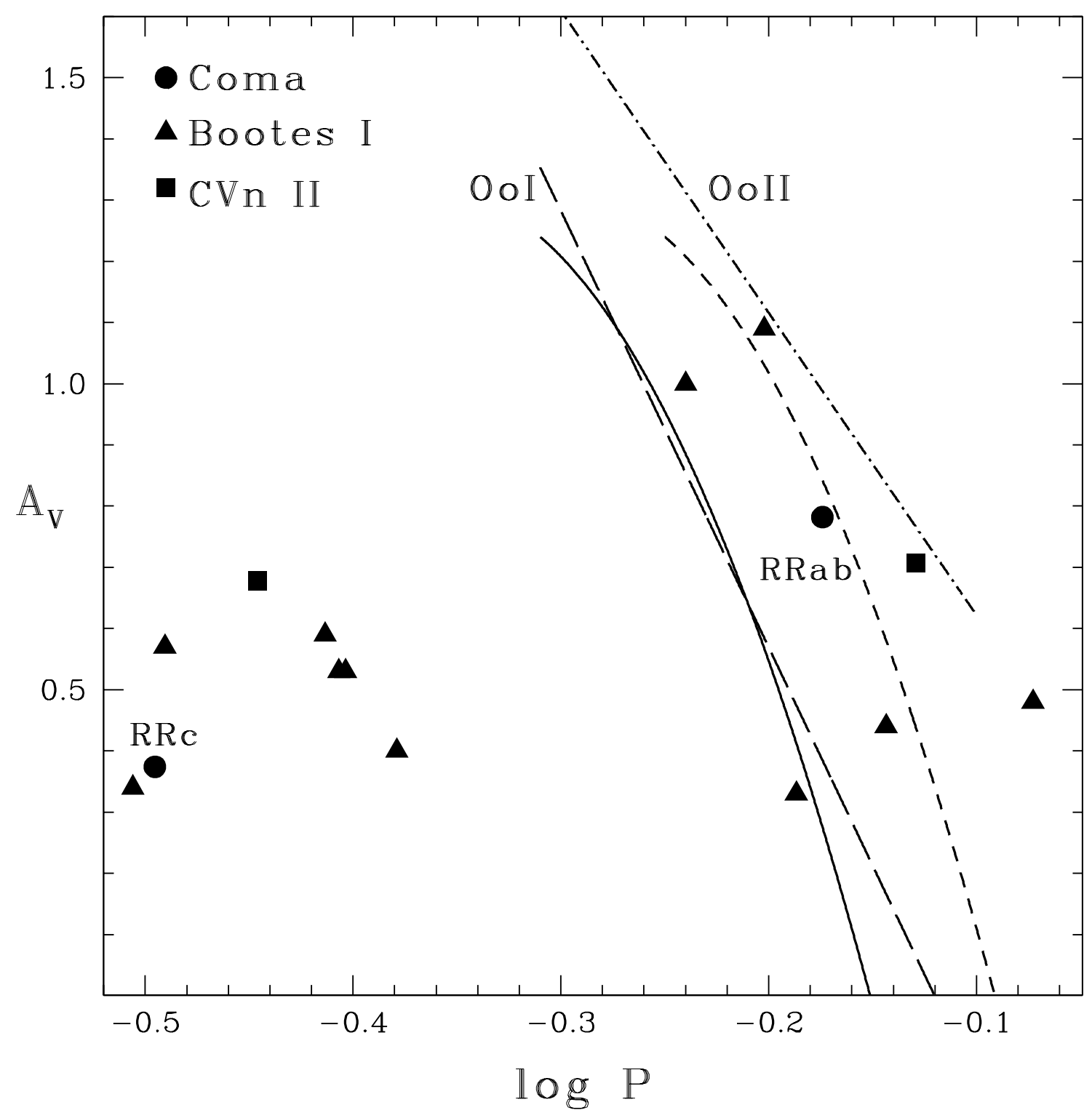

Fig. 2.- Period-amplitude diagram in the $V$ band. Dashed and dotted-dashed lines are the positions of the OoI and Oo II Galactic GCs, from Clement \& Rowe (2000). Periodamplitude distributions of the bona fide regular (solid curve) and well-evolved (dashed curve) fundamental-mode RR Lyrae stars in M3, from Cacciari et al. (2005), are also shown for comparison. 

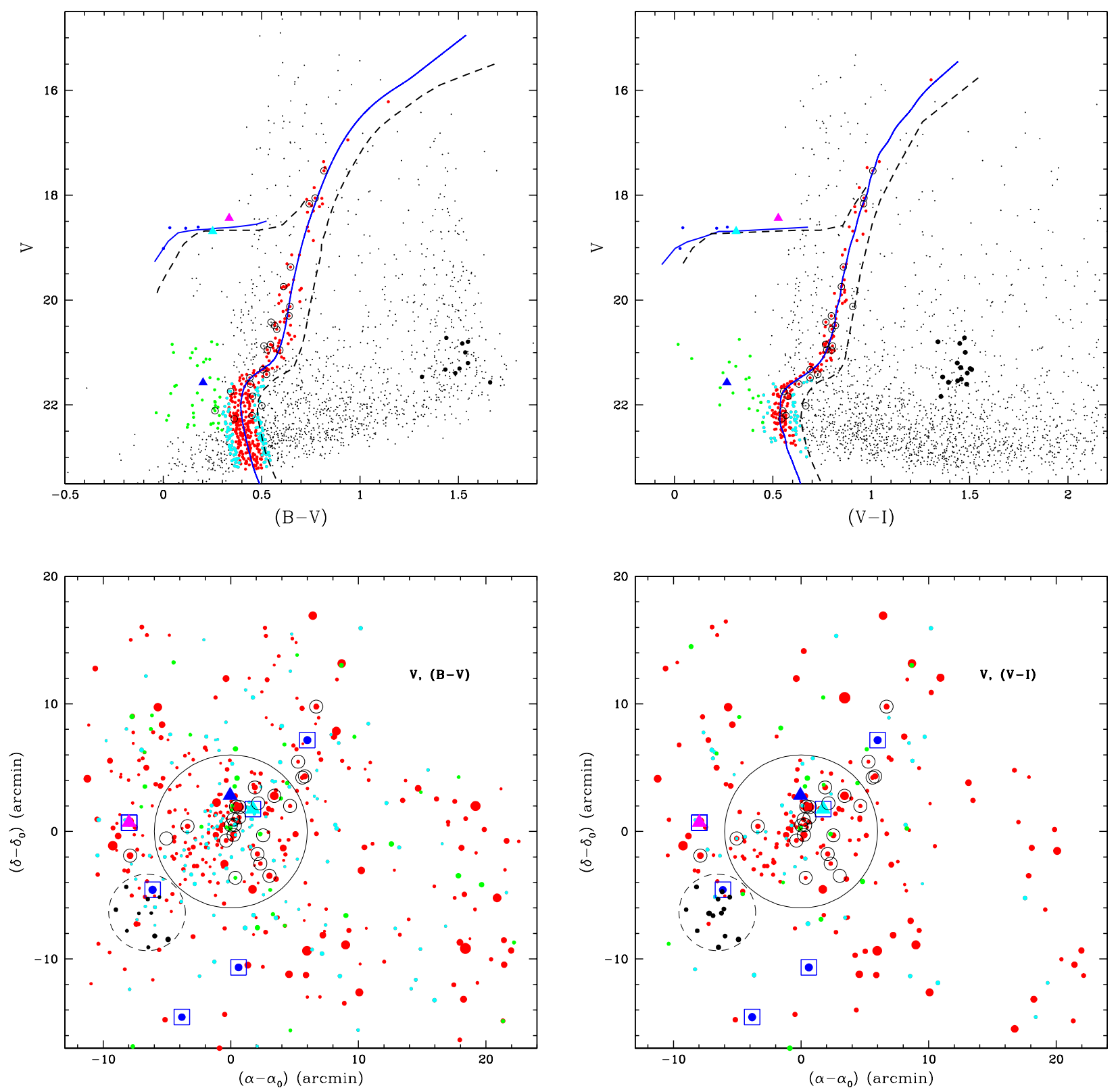

Fig. 3.- Upper panels: $V, B-V$ (left) and $V, V-I$ (right) CMDs of sources in the INT field of view. Solid (blue) and dashed (black) lines are the ridgelines of the Galactic GCs M 68 and M 3, respectively. Red and cyan dots are stars respectively within \pm 0.06 mag in $B-V$ and $\pm 0.05 \mathrm{mag}$ in $V-I$, and from $\pm 0.05 / 0.06$ to $\pm 0.1 \mathrm{mag}$ from the ridgelines of M 68 . Blue dots are non-variable stars on the HB, green dots are stars in the BSSs region. Variable stars are marked by filled triangles, cyan: RRc star, magenta: RRab star, blue: short period variable. Open circles mark 24 member stars of the Coma dSph from Kirby et al. (2008). Black dots are galaxies of cluster MaxBCG J186.85861+2380004. Lower panels: maps of objects most likely belonging to the Coma dSph, color-coded and with same symbols as in the top-panels of the figure. HB stars are outlined by squares. The large circles show the galaxy half-light radius $r_{h}$ (exponential) $=5.9 \operatorname{arcmin}(\mathrm{B} 07)$. The dashed circles show the position of the galaxy cluster. 